\title{
Relation entre dynamique de croissance et dynamique de prélèvement d'azote pour un peuplement de graminées fourragères. I. - Etude de l'effet du milieu
}

\author{
Gilles LEMAIRE \& Jean SALETTE \\ avec la collaboration technique de Monique SIGOGNE \& Jean-Pierre TERRASSON \\ I.N.R.A., Station d'Agronomie, route de Saint-Clément, Beaucouzé, F 49000 Angers et antenne expérimentale, \\ F 86600 Lusignan
}

RÉSUMÉ

\begin{abstract}
Pour un peuplement pur de graminées, en repousse de printemps, et pour diverses doses d'azote, on a étudié les relations entre la croissance, exprimée par l'augmentation de matière sèche récoltable sur des sous-parcelles fauchées successivement au cours d'une repousse, et le prélèvement en azote déterminé par l'azote contenu dans le végétal récolté sur les mêmes sous-parcelles.

L'analyse dynamique du prélèvement d'azote se montre particulièrement intéressante lorsque ce prélèvement est étudié non plus en fonction du temps de repousse mais en fonction de la croissance elle-même (degré de croissance).

En condition de nutrition azotée non limitante, on a pu mettre en évidence une relation d'allométrie valable au cours de la repousse et stable quelle que soit l'année, entre la croissance et les prélèvements en azote. En condition de nutrition azotée limitante, il existe également une relation d'allométrie mais qui varie selon l'année. Les notions de potentiel de croissance et de loi de dilution de l'azote, déjà introduites antérieurement, sont tout particulièrement discutées et précisées.
\end{abstract}

Mots clés additionnels : Potentiel de croissance, degré de croissance, croissance de printemps, facteurs climatiques, fertilisation azotée, teneur en azote, relations d'allométrie, variabilité interannuelle.

Relationship between growth and nitrogen uptake in a pure grass stand. I. - Environmental effects.

\begin{abstract}
The relationship between dry matter (DM) accumulation and N-uptake throughout growth was studied at three nitrogen rates, in a pure grass stand in spring. The value of making such growth studies in terms of DM accumulation instead of time of growth is emphasized. When nitrogen nutrition was not a growth - limiting factor, a stable allometric relation was determined between growth and $\mathrm{N}$-uptake : under these conditions, the relation equation was the same whatever the year. The corresponding altometric equation to lower $\mathrm{N}$-rates did change with the year, i.e., with climatic conditions.

The concepts of potential growth and dilution curves are particularly discussed.
\end{abstract}

Additional key words : Potential growth, accumulated dry matter, spring growth, climatic factors, nitrogen nutrition, nitrogen content, allometric relations, variability between years.

\section{INTRODUCTION}

Les études que nous avons entreprises sur la croissance des peuplements de graminées prairiales nous ont amenés à définir la notion de croissance en condition de nutrition azotée non limitante (LEMAIRE \& SALETTE, 1982).

Dans le cadre d'un contrat de recherches de la CEE (programme protéines végétales : azote et prairie au printemps, $\mathrm{n}^{\circ} 405$ ).
Pour la croissance de printemps, nous avons pu relier ce potentiel de croissance aux principaux paramètres climatiques limitants de cette saison : en premier lieu, la température et, à un degré moindre, le rayonnement (LEMAIRE \& SALETTE, 1981, 1982 ; LEMAIRE et al., 1982a ; PAYEN et al., 1982). Ces résultats, obtenus sur peuplements monospécifiques de graminées, ont été également confirmés sur une prairie naturelle (LEMAIRE et al., 1982b). 
D'autre part, l'analyse de la variation des teneurs en azote des graminées fourragères au cours de leur croissance nous a permis de formuler une loi de dilution de l'azote dans la matière sèche produite (SALETTE \& LEMAIRE, 1981) :

$$
\mathrm{N} \%=\alpha(\mathrm{MS})^{-\beta} .
$$

Cette relation entraîne une liaison plus fondamentale entre la croissance en MS ( $\left.t . \mathrm{ha}^{-1}\right)$ et les prélèvements d'azote ( $\mathrm{N} \exp )$ :

$$
\mathrm{N} \exp (\mathrm{kg} / \mathrm{ha})=10 \alpha \mathrm{MS}^{(1-\beta)}
$$

dans laquelle $(1-\beta)$ représente le coefficient d'allométrie entre prélèvement d'azote et croissance (rapport entre les vitesses relatives) et ( $10 \alpha)$, la quantité d'azote nécessaire à la formation de la première tonne de matière sèche. Ces premières études ont pu montrer que cette relation d'allométrie était en fait déterminée par le niveau de la nutrition azotée, le coefficient $\alpha$ augmentant avec la dose d'azote apportée (SALETTE et al., 1982).

Dans ces conditions il est important de savoir si, pour un niveau de nutrition azotée donné, cette relation entre croissance et prélèvement d'azote est stable d'une année à l'autre. Etant donné la difficulté de reproduire au champ des niveaux de nutrition azotée identiques du fait de l'importance incontrôlable de la minéralisation du sol, nous avons essayé de reproduire un niveau de nutrition considéré comme non limitant (c'est-à-dire permettant l'expression du potentiel de croissance permis par le climat) par un apport suffisamment important pour annuler l'effet du sol.

Dans ce premier article nous analyserons la stabilité des relations entre la croissance et les prélèvements d'azote pour un même génotype à travers des milieux climatiques variables représentés par des années différentes en un même lieu, et nous essayerons de dégager la part de la fourniture d'azote par le sol.

\section{MATÉRIELS ET MÉTHODES}

Les résultats développés ici ont été obtenus dans 2 expérimentations sur des croissances de printemps :

\section{Expérimentation 1 :}

L'analyse de la croissance au printemps a été réalisée sur des peuplements de Festuca arundinacea (cv. "Ludelle ») installés par semis, au printemps 1974, à Lusignan. Deux niveaux de nutrition azotée ont été utilisés : un niveau faible: $60 \mathrm{~kg} \mathrm{~N}$.ha ${ }^{-1}$ apportés à l'automne ; un niveau élevé considéré comme non limitant : $60 \mathrm{~kg} \mathrm{~N}$. ha ${ }^{-1}$ apportés à l'automne, suivis d'un apport de $120 \mathrm{~kg} \mathrm{~N} \cdot \mathrm{ha}^{-1}$ en fin d'hiver (mi-février). La croissance de printemps de la graminée a été suivie en 1975, 1976 et 1977 par des prélèvements hebdomadaires à la motofaucheuse (hauteur de coupe $5 \mathrm{~cm}$ ) sur des parcelles élémentaires d'une surface de $6 \mathrm{~m}^{2}$. Nous avons réalisé à chaque printemps de 10 à 12 coupes successives pour décrire la courbe de croissance depuis le départ en végétation jusqu'à l'épiaison.

A chacune des coupes successives, effectuées sur les parcelles élémentaires adjacentes, des échantillons de la partie récoltée étaient prélevés pour analyse des teneurs en azote, ce qui permet de calculer les quantités d'azote prélevées par la graminée dans ses parties récoltables (SALETTE et al., 1982).

\section{Expérimentation 2 :}

Un essai du même type a été mis en place au printemps 1979 à Lusignan, comportant 3 génotypes différents :

Festuca arundinacea cv. "Ludelle» cv. "Clarine" cv. «EM 77 » (variété expérimentale résultant du croisement entre fétuques européenne et méditerranéenne avec restauration de la fertilité par doublement des chromosomes).

Dans cet essai, l'objectif était de comparer les dynamiques de prélèvement d'azote entre les différents génotypes, en condition de nutrition azotée non limitante. Pour nous assurer d'avoir effectivement réalisé cette condition, nous avons appliqué 2 niveaux d'apport : l'un correspondant au niveau non limitant de l'expérimentation 1, c'est-à-dire $60 \mathrm{~kg} \mathrm{~N} \cdot \mathrm{ha}^{-1}$ à l'automne, plus $120 \mathrm{~kg} \mathrm{~N}$. ha ${ }^{-1}$ en fin d'hiver ; l'autre, plus élevé de $30 \mathrm{~kg} / \mathrm{ha}: 60 \mathrm{~kg} \mathrm{~N}$. ha ${ }^{-1}$ à l'automne, plus $150 \mathrm{~kg} \mathrm{~N}$. ha ${ }^{-1}$ en fin d'hiver. L'absence de différence significative de croissance entre ces 2 niveaux permet d'affirmer que le niveau $60+120$ correspondait bien à ces conditions non limitantes de nutrition azotée.

La croissance et les prélèvements d'azote des différents génotypes ont été suivis durant les printemps 1980,81 et 82 selon la même méthode que celle utilisée dans l'expérimentation 1.

\section{Conditions générales de l'essai}

Le type de sol localement appelé «terres rouges à châtaigniers » est en fait un sol brun lessivé sur limon, superposé à une argile rouge à pisolithes. Il ne présente aucune caractéristique pouvant avoir un effet limitant sur la croissance ou l'absorption d'azote. Une fumure P K largement suffisante a été apportée lors de l'installation, complétée chaque année d'apports en couverture restituant largement les exportations.

Le climat des différentes années et son effet direct sur la croissance ont été analysés en détail dans des notes précédentes (LEMAIRE \& SALETTE, 1982; LEMAIRE et al., 1982a). Le tableau 1 en résume les caractéristiques principales.

\section{RÉSULTATS}

Dans cette note, nous analysons d'une part la variabilité entre années des relations entre les dynamiques de croissance et les dynamiques de prélèvement d'azote sur " Ludelle ", à travers les 2 séries d'expérimentation, en utilisant les données du niveau de fertilisation azotée considéré comme non limitant. D'autre part, nous analysons les prélèvements d'azote pour les niveaux faibles dans les années 1975, 76, 77, afin de mettre en évidence la part de l'azote fourni par le sol.

\section{A. Dynamique de prélèvement en condition de nutri- tion azotée non limitante}

L'analyse des courbes de croissance obtenues sur la fétuque élevée "Ludelle " en condition d'azote non 
TABLEAU 1

Températures moyennes décadaires de l'air à Lusignan (sous abri) Mean temperatures over ten-day periods at Lusignan



limitant et représentées sur la figure 1 a donné lieu à une série de notes dont les conclusions essentielles sont les suivantes :

- La croissance de printemps doit être décomposée en 2 phases : une phase végétative lors de laquelle la biomasse récoltable ne contient que des feuilles ; une phase reproductrice durant laquelle apparaissent et se développent des tiges. Cette $2^{\mathrm{e}}$ phase repérée en pratique par un stade agronomique dit "épi à $10 \mathrm{~cm}$ » (GILLET, 1980) débute lorsque la majorité des apex ont atteint le niveau de la coupe : ce stade a une signification agronomique et pratique, plus que physiologique, puisque l'initiation des tiges lui est très antérieure. II s'ensuit une augmentation de la vitesse de croissance (visible sur la fig. 1) due au développement des tiges, phase qui s'accompagne en outre d'une augmentation des capacités photosynthétiques du couvert végétal (WOOLEDGE, 1978).

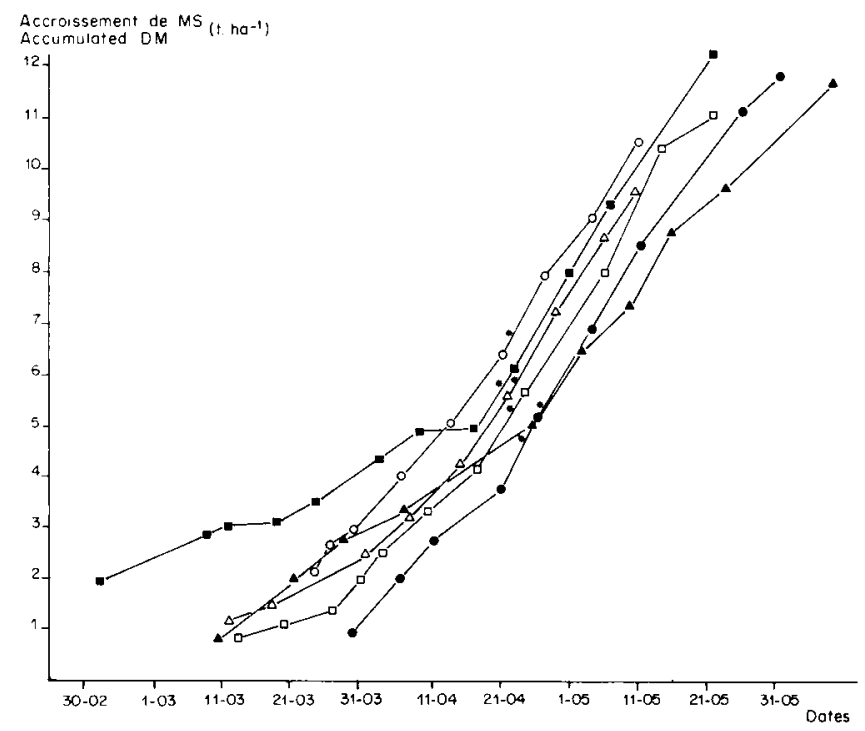

Figure 1

Croissance de printemps de la fétuque élevée "Ludelle " pour une nutrition azotée non limitante, exprimée par l'accroissement de la matière sèche récoltable (MS, $t . h a^{-1}$ ).

Expérimentation (1) : 1975 — $1976 \bullet 1977$ А

Expérimentation (2) : $1980 \square 1981 \bigcirc 1982 \triangle$

*: stade " épi à $10 \mathrm{~cm}$ ».

Spring growth of tall fescue under conditions of non-limiting nitrogen supply, expressed as accumulated herbage dry matter (DM t. $h a^{-1}$ ).

Experiment (1) : $1975 \square 1976 \bullet 1977 \Delta$
Experiment (2) : $1980 \square \quad \square$
$*: 10 \mathrm{~cm}$ ear height.
- La croissance végétative est extrêmement variable en termes de vitesse et en termes de précocité de départ en croissance. Nous avons pu établir que la température était le facteur explicatif essentiel de cette variabilité et une relation linéaire entre la matière sèche récoltée et la somme de température a pu être proposée.

- La croissance reproductrice est par contre très stable en vitesse et les courbes de croissance restent parallèles à partir du stade épi à $10 \mathrm{~cm}$, excepté pour l'année 1977 qui présente une croissance légèrement plus faible.

L'analyse des courbes de prélèvements d'azote (fig. 2) met en évidence une variabilité très importante, à la fois durant la phase végétative, mais également durant la phase reproductrice : ainsi, au $1^{\text {er }}$ avril, le peuplement de graminées avait prélevé environ $50 \mathrm{~kg}$ d'azote en 1976 contre 120 environ en 1975. Cet écart très important est à rapprocher des différences constatées dans la croissance à cette date : environ $1 \mathrm{t}$ de MS en 1976 contre 4 en 1975 . Les prélèvements maximum

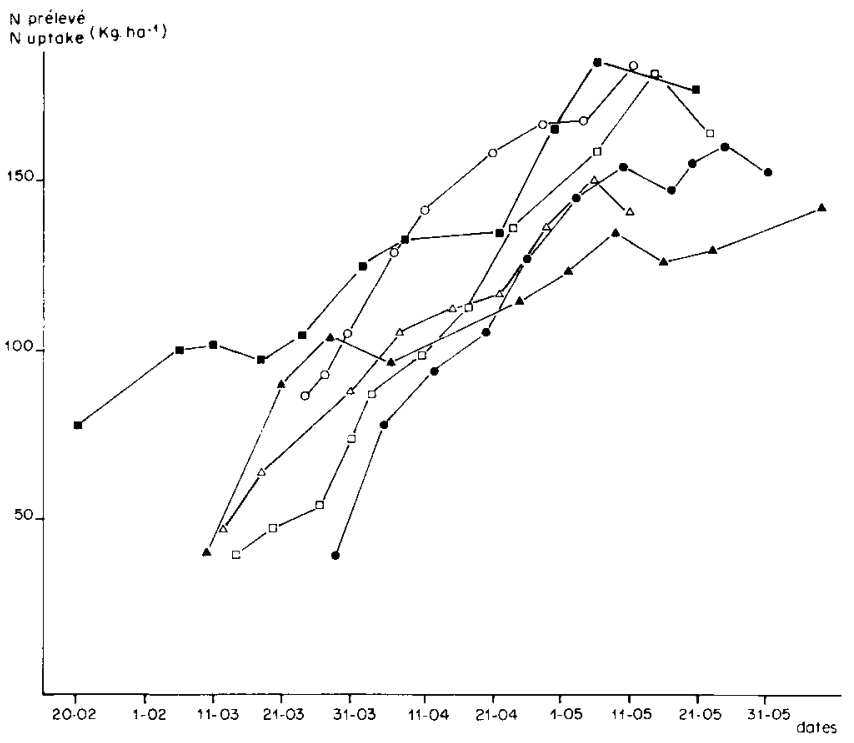

Figure 2

Prélèvements d'azote de la fétuque élevée "Ludelle» pour une nutrition azotée non limitante, au cours de la croissance de printemps ( $\mathrm{kg} \mathrm{N} . \mathrm{ha} \mathrm{C}^{-1}$ ).

Mêmes symboles que pour la figure 1 .

Nitrogen uptake of tall fescue for a non-limiting nitrogen supply, throughout the spring growth ( $\mathrm{kg} \mathrm{N} . \mathrm{ha}^{-1}$ ).

Same symbols as for fig. 1 . 
d'azote en fin de croissance varient de $130 \mathrm{~kg}$ environ en 1977 à $180 \mathrm{~kg}$ en 1975,1980 et 1981 ; on peut être tenté de voir là une des raisons de la plus faible croissance observée en phase reproductrice en 1977.

Lorsque l'on analyse la dynamique des prélèvements d'azote, non plus en fonction du temps, mais en fonction de la matière sèche accumulée (fig. 3a), on s'aperçoit que les écarts très importants observés en début de printemps sont entièrement expliqués par des différences concomitantes de la croissance. L'analyse des données en coordonnées logarithmiques (fig. 3 b) permet de définir une relation allométrique unique entre production de matière sèche et prélèvements d'azote pour la première partie de la croissance des différentes années. L'examen graphique des données nous permet de mettre en évidence une cassure très nette de la pente de la relation d'allométrie en fin de croissance pour chacune des 6 années d'étude.

Nous avons pu repérer graphiquement pour chaque année les points au-delà desquels la relation d'allométrie initiale ne semblait plus vérifiée. Le tableau 2 donne pour chacun de ces points : la date correspondante, la quantité de matière sèche formée, et la quantité d'azote prélevée.

Il ressort des données de ce tableau que la diminution du coefficient d'allométrie en fin de croissance ne semble pas correspondre à un changement morphologique du couvert végétal dû au démarrage de la croissance des tiges puisque les dates correspondantes sont très variables d'une année sur l'autre (du 2 avril au 20 avril), alors que la date du stade " épi à $10 \mathrm{~cm}$ » est relativement fixe ( 20 au 25 avril) parce qu'elle dépend en grande partie de la longueur du jour.

D'autre part, on peut noter que ce ralentissement du prélèvement d'azote relativement à la croissance ne correspond ni à une quantité donnée d'azote prélevé, ni à une quantité donnée de matière sèche élaborée. Il ne semble donc pas que l'on doive rechercher dans les caractéristiques du couvert végétal lui-même la cause de cette diminution.

L'interprétation que nous proposons consiste à admettre que la relation allométrique initiale $\mathrm{N} \exp =47,9$ (MS) ${ }^{0,676}$ représente une loi générale décrivant la dynamique des prélèvements d'azote par rapport à la croissance, avec une offre en azote non limitante de la part du milieu.

\section{TABLEAU 2}

Coordonnées du point singulier au-delà duquel la relation d'allométrie initiale entre croissance et prélèvements d'azote pour les différente: années n'est plus identique quelle que soit l'année (fig. $3 b$ ).

Characteristics of the point beyond which the initial allometric relation between $N$ uptake and growth is no longer the same for the different years (fig. $3 b$ ).

\begin{tabular}{lrcr}
\hline \hline & $\begin{array}{c}\text { Date de } \\
\text { réalisation }\end{array}$ & MS t.ha ${ }^{-1}$ & N exp kg.ha ${ }^{-1}$ \\
\hline 1975 & 2.04 & 4,35 & 125 \\
1976 & 20.04 & 3,90 & 115 \\
1977 & 6.04 & 3,30 & 98 \\
1980 & 10.04 & 3,30 & 100 \\
1981 & 6.04 & 4,90 & 140 \\
1982 & 7.04 & 3,40 & 105 \\
\hline
\end{tabular}
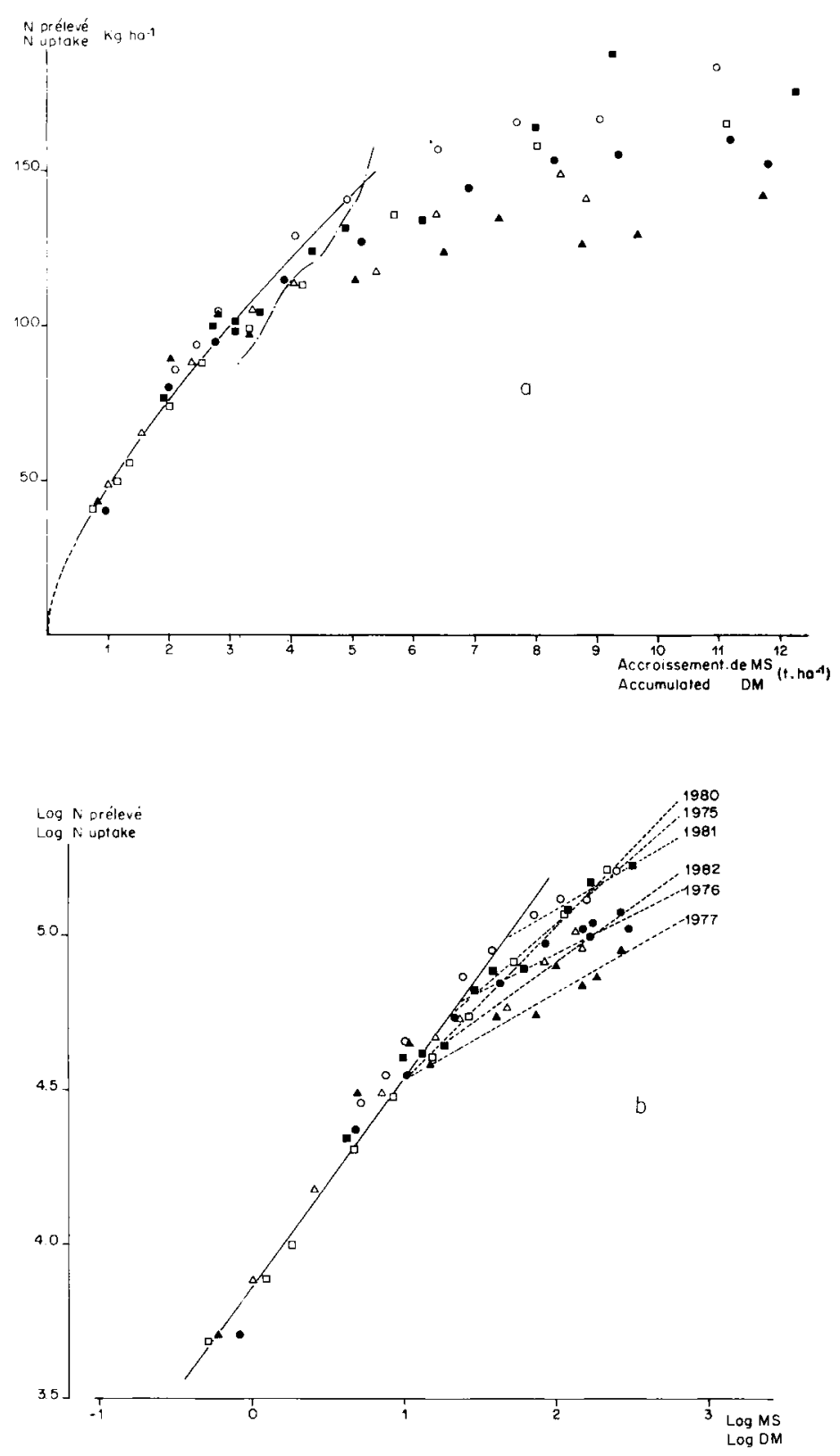

Figure 3

Relation allométrique entre le prélèvement d'azote $\left(\mathrm{kg} \mathrm{N}^{\mathrm{N}} \mathrm{ha^{-1 }}\right.$ ) et la croissance exprimée par l'accroissement de la matière sèche récoltable (MS, $1 . h a^{-1}$ ), fétuque élevée "Ludelle "; nutrition azotée non limitante.

Mêmes symboles que pour la figure 1.

$3(a): N \exp =47,9(M S)^{+0,676} r=+0,981$

3 (b) $: \log N \exp =0,676 \log M S+3,87$

-.-. : Séparation des points non pris en compte dans la régression.

Allometric relation between nitrogen uptake and accumulated herbage dry matter $\left(t . h a^{-1}\right)$. Tall fescue $c v$. "Ludelle", non-limiting $N$ supply.

Same symbols as fig. 1 .

$3\left(\right.$ a) : N upt $=47,9(D M)^{+0,676} r=+0.981$

3 (b) : Log N upt $=0.676 \log D M+3.87$

-._.- : Points beyond this line not taken into account in regression.

Dans les conditions du champ, l'offre en azote du milieu a tendance à diminuer avec le temps du fait des prélèvements eux-mêmes, mais aussi éventuellement du fait de phénomènes de réorganisation de l'azote minéral par la biomasse microbienne du sol (SALETTE \& LEMAIRE, données non publiées). Cette diminution de 
l'offre en $\mathrm{N}$ du milieu peut être elle-même atténuée par des minéralisations plus ou moins importantes en fin de printemps. Il s'ensuit une nouvelle relation allométrique entre prélèvements d'azote et croissance, caractéristique de cette phase d'épuisement de l'azote disponible du milieu. Nous avons pu déterminer ces relations pour les différentes années :

$\begin{array}{lll}1975 & \mathrm{~N} \exp =66,0(\mathrm{MS})^{0,426} & \mathrm{r}=0,967 \\ 1976 & \mathrm{~N} \exp =80,6(\mathrm{MS})^{0,286} & \mathrm{r}=0,950 \\ 1977 & \mathrm{~N} \exp =70,8(\mathrm{MS})^{0,281} & \mathrm{r}=0,929 \\ 1980 & \mathrm{~N} \exp =54,6(\mathrm{MS})^{0,519} & \mathrm{r}=0,998 \\ 1981 & \mathrm{~N} \exp =90,9(\mathrm{MS})^{0,293} & \mathrm{r}=0,972 \\ 1982 & \mathrm{~N} \exp =67,4(\mathrm{MS})^{0,355} & \mathrm{r}=0,947\end{array}$

On peut remarquer que les années 1975 et 1980 , qui correspondent en fait à la première année suivant l'année de semis de la fétuque pour chaque série d'expérimentation, sont celles qui conservent en fin de printemps le coefficient d'allométrie le plus élevé. On pourrait interpréter cela par l'hypothèse, à vérifier, que l'accumulation de matières organiques riches en carbone (vieilles racines) au cours de vieillissement d'une prairie conduirait à une augmentation de la compétition entre la biomasse microbienne du sol et la plante pour l'utilisation de l'azote minéral. Ceci repose le problème du comportement différent du peuplement prairial, d'une part, en fonction de l'âge du peuplement, la $1^{\text {re }}$ année étant le plus souvent très différente, d'autre part, en fonction des changements dans la dynamique de l'azote dans le système plante-sol.

\section{B. Dynamique de prélèvement en absence d'apport d'azote au printemps}

L'examen des dynamiques de prélèvement d'azote et des dynamiques de croissance pour la dose faible en 1975, 1976 et 1977 (fig. 4) nous permet de rendre compte de la très grande variabilité interannuelle de la fourniture d'azote par le sol. L'expression des prélèvements d'azote en fonction de la matière sèche permet de mettre en relief les différences de disponibilité en azote entre les 3 années (fig. 5). Cette disponibilité peut être mesurée, en début de croissance, par la quantité d'azote utilisée par le couvert végétal pour l'élaboration de la première tonne de matière sèche : $43,4 \mathrm{~kg}$ en 1975 , $21,6 \mathrm{~kg}$ en 1976 et $28,6 \mathrm{~kg}$ en 1977 . Ceci est à rapprocher des $46,7 \mathrm{~kg}$ obtenus avec le niveau d'azote non limitant.

\section{DISCUSSION}

\section{A. Relation d'allométrie entre prélèvement d'azote et croissance}

L'équation (2), citée plus haut, peut s'écrire :

$$
\log (\mathrm{N} \exp )=(1-\beta) \log (\mathrm{MS})+\log (10 \alpha)
$$

et en dérivant par rapport au temps ;

$$
\frac{\mathrm{dN} \exp }{\mathrm{N} \exp \cdot \mathrm{dt}}=(1-\boldsymbol{\beta}) \frac{\mathrm{dMS}}{\mathrm{MS} \cdot \mathrm{dt}} \text {. }
$$

Le coefficient $(1-\beta)$ doit être interprété comme le rapport entre les vitesses relatives de prélèvement
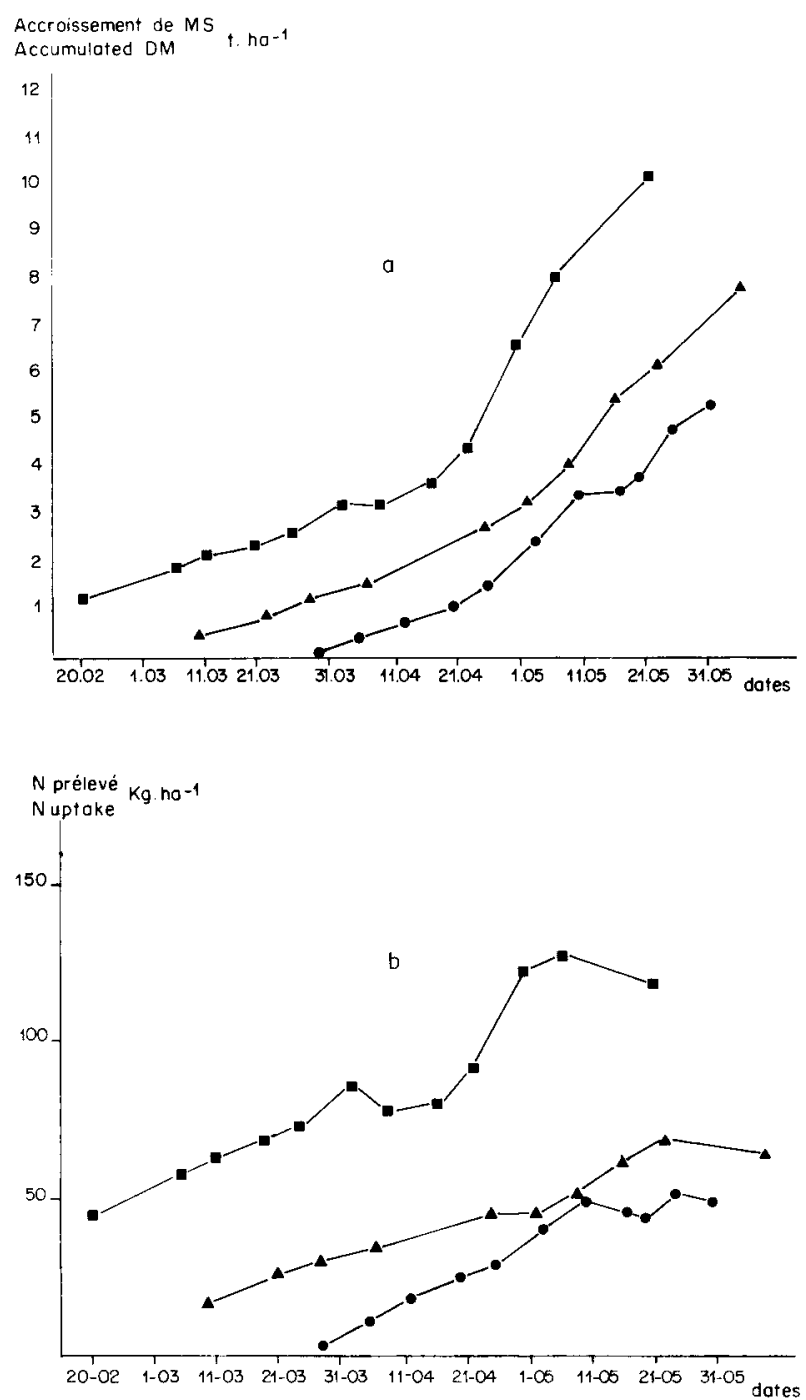

Figure 4

Croissance (a) et prélèvements d'azote (b) de la fétuque élevée «Ludelle » au printemps, à faible niveau de nutrition azotée.

$$
1975 \bullet 1976 \triangle 1977
$$

Spring growth of tall fescue (a) and nitrogen uptake (b), for a limited $N$ supply.

$1975 \bullet 1976 \triangle 1977$

d'azote et de croissance en matière sèche. Ou bien encore :

$$
\frac{d N \exp }{d M S}=(1-\beta) \frac{N \exp }{M S} .
$$

En condition d'offre en azote non limitant, l'équation (5) permet d'exprimer les besoins instantanés d'azote par $\mathrm{kg}$ de matière sèche élaborée. On voit donc que le fait que (1- $\beta$ ) soit inférieur à 1 implique un amortissement progressif des besoins en azote au cours de la croissance pour l'élaboration de la matière sèche. Ceci se traduit, au niveau de la plante, par une " dilution" de l'azote par la matière sèche élaborée. Cette dilution $s$ 'exprime par la relation inverse :

$\mathrm{N} \%=\alpha(\mathrm{MS})^{-\beta}$ (1) déjà rappelée dans l'introduction de cette note.

Cet amortissement des besoins en azote de la plante par rapport à sa croissance peut s'expliquer de 2 manières :

D'une part, en début de repousse, la croissance est 


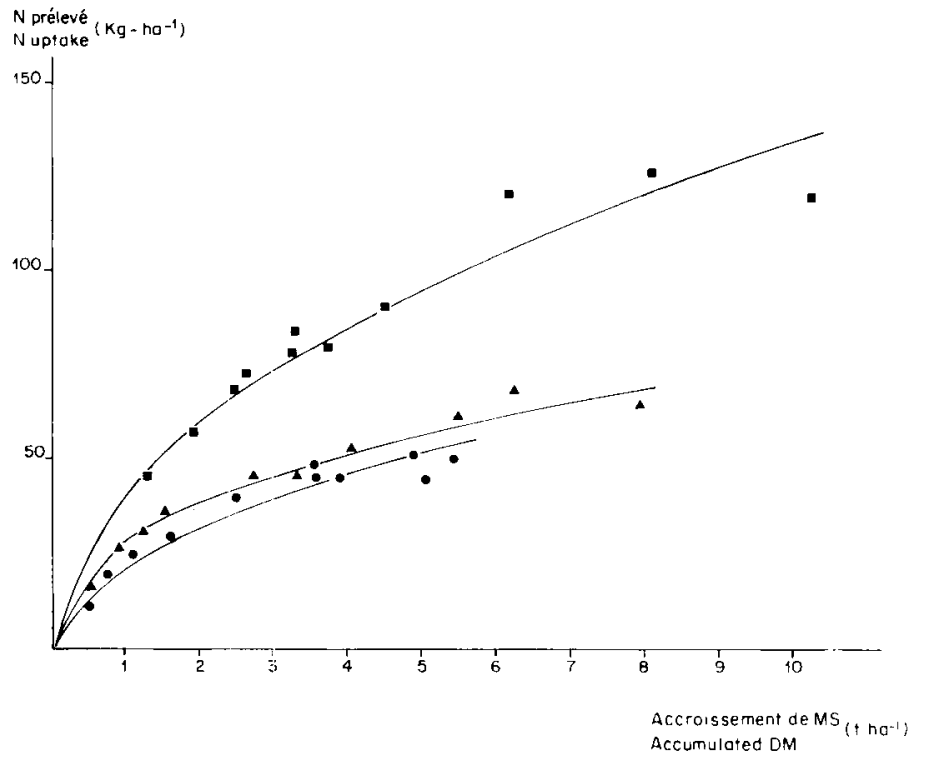

Figure 5

Relations d'allométrie entre prélèvements d'azote et croissance de la fétuque élevée "Ludelle» en absence de fumure azotée au printemps.

$1975 N \exp =43,4(M S)^{0,498}$

- $1976 N$ exp $=21,6(M S)^{0,551}$

- $1977 \quad N$ exp $=28,6(M S)^{0,411}$

Allometric relation between nitrogen uptake and growth of tall fescue, for a limited $N$ supply

$1975 N u p t=43,4(D M)^{0.498}$

- $1976 N u p t=21,6(D M)^{0.551}$

- $1977 N u p=28,6(D M)^{0.411}$.

assurée principalement par la morphogenèse et l'élongation de nouveaux organes (jeunes feuilles et jeunes tiges) ; cette phase nécessite essentiellement des protéines, les besoins en azote par $\mathrm{kg}$ de matière sèche élaborée sont alors élevés. Le déploiement de ces organes va permettre une augmentation de la masse de carbone fixée conduisant à la différenciation des tissus et une augmentation rapide de leur poids par unité de surface (GILlET et al., 1984). D'autre part, les feuilles ayant une durée de vie relativement courte (4 à 6 semaines, selon GILLET, 1980), il existe des mécanismes de recyclage des protéines à partir des feuilles sénescentes, permettant à la plante de continuer à fabriquer de nouvelles générations de feuilles (THOMAS \& STODDART, 1980).

Compte tenu de ces 2 observations, il serait intéressant de savoir .usqu'à quelle limite la relation allométrique initiale pourrait se poursuivre si l'on maintenait l'offre du milieu constante par un apport supplémentaire d'azote en cours et en fin de croissance. S'agissant d'un cycle reproducteur, l'importance croissante du poids des tiges impliquerait à elle seule un amortissement des besoins en azote plus rapide que ne le laisse supposer le modèle initial établi pour une période végétative. Des études en culture en milieu contrôlé, avec maintien d'une nutrition azotée à offre constante pendant toute la croissance seraient nécessaires pour préciser les bases physiologiques de ce modèle. Une analyse simultanée du rapport feuilles/tiges serait en outre très instructive (SALETTE \& DUMAS, 1970).

\section{B. Effet du sol}

Quoi qu'il en soit, l'amortissement plus ou moins marqué des exportations d'azote en fin de cycle semble, dans nos conditions, être nettement explicable par une diminution de l'offre en azote du milieu ; il se produit, certaines années, bien avant le début d'apparition des tiges.

L'examen des dynamiques d'absorption permises par la seule fourniture d'azote par le sol (niveau faible en 1975,76 et 77 ) permet de dégager un certain parallélisme en fin de croissance avec les niveaux non limitants. En effet, si on calcule les prélèvements d'azote en $\mathrm{g}$ de $\mathrm{N}$ par $\mathrm{kg}$ de MS élaborée grâce à l'équation (5) pour la fin de la croissance on obtient des chiffres relativement voisins entre les 2 doses $N_{0}$ et $N_{2}$ d'une même année et variant du simple au double entre 1977 et 1975 (tabl. 3). Le prélèvement plus faible obtenu en 1976 sur le niveau non limitant en azote peut être attribué à la sécheresse exceptionnelle de cette fin de printemps.

Il semble que la dynamique de prélèvement d'azote de la prairie avec la dose limitante se soit ajustée plus ou moins rapidement, selon les années, à la fourniture d'azote par le sol.

\section{Conséquences sur la croissance en matière sèche}

Le potentiel de croissance ayant été atteint pour les différentes années, excepté peut-être en 1977 (cf. fig. 1), il semble bien que les différentes consommations d'azote constatées en fin de croissance entre les années aient dû s'accompagner soit d'une différence de répartition de la matière sèche entre les feuilles et les tiges, soit (et) d'une différence d'intensité dans les phénomènes de recyclage. A cet égard, il serait intéressant de contrôler les formes d'azote $\left(\mathrm{NO}_{3}^{-}\right.$, protéines solubles, protéines insolubles) durant la première partie de la croissance. Un stockage important d'azote dans l'appareil foliaire durant cette phase initiale pourrait expliquer l'absence de réponse ultérieure de la croissance en matière sèche aux différences de vitesse de prélèvement d'azote.

\section{Loi de dilution de l'azote au cours de la croissance}

La figure 6 représente la dilution de l'azote dans la matière sèche élaborée au cours de la croissance. Pour chaque année, on peut en formuler une expression mathématique conformément à l'équation (1) (SALETTE \& LEMAIRE, 1981).

\section{TABLEAU 3}

Quantités d'azote prélevées par $\mathrm{kg}$ de MS élaborée en fin de croissance en 1975, 1976 et 1977 (en $\mathrm{g} N$ par $\mathrm{kg}$ de MS).

Nitrogen uptake ( $g N h^{-1}$ per $k g$ of accumulated $D M . h a^{-1}$ ) at the last phase of growth.

\begin{tabular}{llll}
\hline & 1975 & 1976 & 1977 \\
\hline$N_{0}$ & 6,70 & 5,50 & 3,42 \\
$N_{2}$ & 6,85 & 4,23 & 3,49 \\
\hline \hline
\end{tabular}




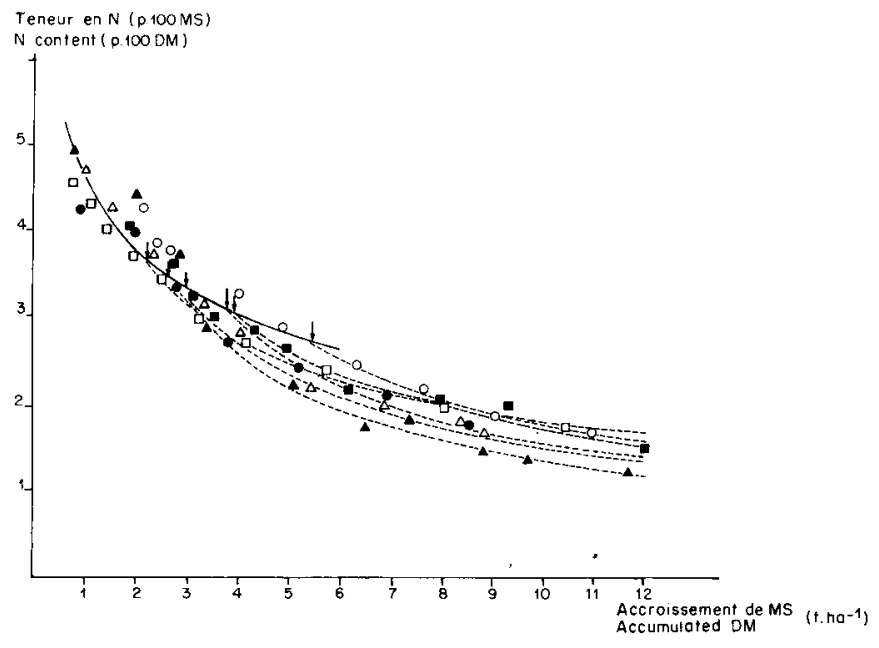

Figure 6

Courbes de dilution de l'azote dans la matière sèche élaborée au cours de la croissance de printemps de la fétuque élevée "Ludelle" $(N \%=f(M S))$.

$\begin{array}{ll}\text { - } 1975 & \square 1980 \\ -1976 & \bigcirc 1981 \\ \Delta 1977 & \triangle 1982\end{array}$

Courbe de dilution initiale commune aux 6 années : $N \%=4,79(M S)^{-0,324}$

--- Courbe de dilution à partir du point singulier ( $\downarrow)$ :

$1975: N \%=6,60(M S)^{-0,574}$

$1976: N \%=8,06(M S)^{-0,714}$

$1977: N \%=7,08(M S)^{-0,719}$

$1980: N \%=5,46(M S)^{-0,481}$

$1981: N \%=9,09(M S)^{-0,707}$

$1982: N \%=6,73(M S)^{-0,645}$

Curve of nitrogen dilution in accumulated dry matter throughout spring growth for tall fescue cv. "Ludelle" $[N \%=f(D M)]$ for 6 different years :

— Initial dilution curve identical for the 6 studied years.

-.-.- Dilution curve beyond the particular point (1).

Tant que la disponibilité en azote reste non limitante, généralement dans la phase initiale de la croissance, on retrouve une dynamique de dilution commune aux différentes années. La différenciation entre années intervient en fin de croissance et correspond à l'épuisement de l'azote disponible. Il est donc difficile de proposer, pour l'ensemble de la croissance d'une repousse, une formulation mathématique rigoureuse et unique de cette loi de dilution. Cependant, l'examen de la figure 1 montre qu'en fin de croissance l'ensemble des courbes restent remarquablement parallèles, excepté l'année 1977 qui présente un net fléchissement. On peut rapprocher cette croissance plus faible de 1977 de la dilution plus prononcée de l'azote qui est un indice, dans ce cas, de faible disponibilité de l'azote dans le milieu.

Il paraît donc possible de déterminer la courbe de dilution au-dessous de laquelle la disponibilité en azote deviendrait un facteur limitant de la croissance en matière sèche.

Une estimation approchée de cette courbe limite de dilution peut toutefois être proposée en calculant une relation de dilution uniforme pour l'ensemble des années (excepté 1977) 'et en négligeant la rupture de pente des relations d'allométrie : la figure 7 représente cette relation de référence, avec azote non limitant, par rapport à laquelle sont placées les relations de dilution obtenues en 1975, 76 et 77, sans apport d'azote au

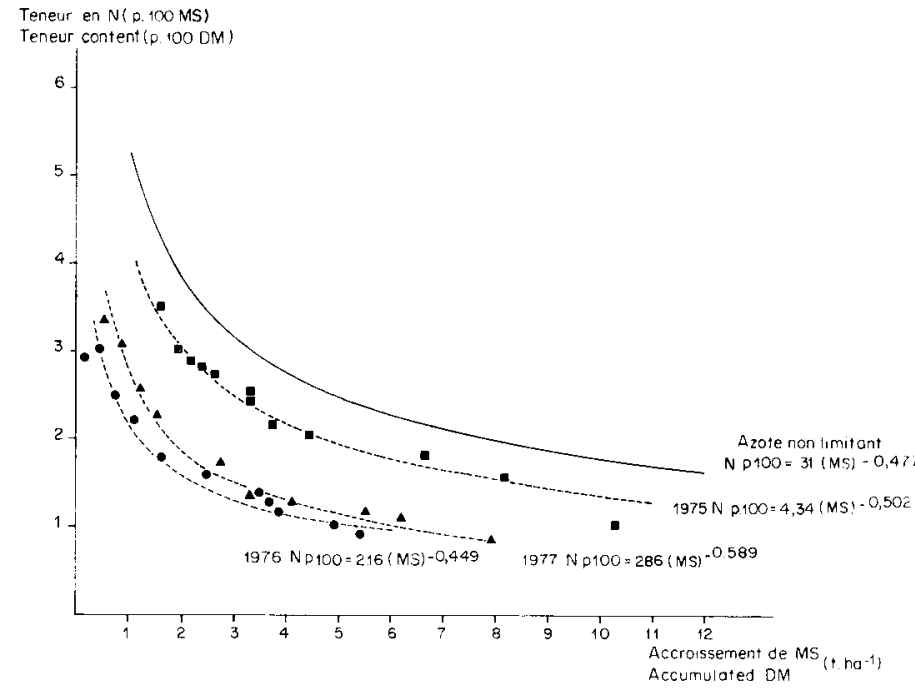

Figure 7

Courbes de dilution de l'azote dans la matière sèche élaborée au cours de la croissance de printemps de la fétuque élevée "Ludelle" $(N \%=f(M S))$.

$\frac{C o u r b e}{(M S)^{-0,477}}$ de référence, azote non limitant: $N \%=5,31$ Court

---- Courbe de dilution sans apport d'azote au printemps.

- $1975 ; \bullet 1976 ; \boldsymbol{\Delta} 1977$

$1975: N \%=4,34(M S)^{-0,502}$

$1976: N \%=2,16(M S)^{-0,449}$

$1977: N \%=2,86(M S)^{-0,589}$

Curves of nitrogen dilution in accumulated dry matter throughout spring growth for tall fescue cv. "Ludelle" $[N \%=f(D M)]$ :

- Reference curve for non-limiting $N$ supply

---- Actual dilution curve without extra nitrogen application in spring.

printemps. La distance de ces courbes à la courbe de dilution de référence peut être interprétée comme une mesure du déficit de l'offre en azote du milieu. Ainsi, un diagnostic sur le niveau de nutrition azotée d'une prairie peut-il être fait par simple positionnement de sa production de matière sèche et de la teneur en azote correspondante par rapport à une courbe de référence.

\section{CONCLUSIONS}

Les résultats obtenus pendant 6 années d'essais confirment l'intérêt qu'il y a à exprimer la dynamique de prélèvement d'azote d'une prairie par rapport à sa croissance en matière sèche (SALETTE \& LEMAIRE, 1981). Pour une offre donnée du milieu en azote, il semble bien qu'il y ait un ajustement réciproque des vitesses de croissance et de prélèvement d'azote. En conditions d'offre en azote non limitantes (la vitesse de croissance est alors seulement limitée par le climat instantané), on peut définir les relations causales suivantes ;

(1) climat $\rightarrow$ potentiel de croissance

(2) potentiel de croissance $\rightarrow$ prélèvements d'azote.

Cette relation (2) semble très stable d'une année sur l'autre tant que l'offre en azote du milieu reste suffisante.

Dans un sol de prairie, l'offre en azote du sol, maximum juste après un apport d'engrais, s'épuise au fur et à mesure des prélèvements et de la réorganisation et s'aligne plus ou moins rapidement sur celle des parcelles non fertilisées. 
Du point de vue pratique, il apparaît important que la prairie puisse profiter au maximum de cet enrichissement momentané du milieu ; sa capacité de prélèvement instantané étant elle-même dépendante de son potentiel de croissance, ceci pose le problème de la date d'apport d'azote, notamment au printemps.

La comparaison entre génotypes différents dans un même milieu, analysée dans une note suivante, nous à permis d'approfondir l'étude des relations entre prélèvements d'azote et croissance : les différents génotypes permettent en effet une variation du potentiel de croissance indépendamment des conditions climatiques qui peuvent elles-mêmes avoir des répercussions sur la disponibilité de l'azote du sol.

Recu le 13 mai 1983 Accepté le 12 janvier 1984.

\section{RÉFÉRENCES BIBLIOGRAPHIQUES}

Gillet M., 1980. Les graminées fourragères. Gauthier-Villars éd., Paris, $306 \mathrm{p}$

Gillet M., Lemaire G., Gosse G., 1984. Essai d'élaboration d'un schéma global de la croissance des graminées fourragères. Agronomie, 4 (1), 75-82.

Lemaire G., Salette J., 1981. Analyse de l'influence de la température sur la croissance de printemps de graminées fourragères. C.R. Acad. Sci. Paris, 292, 843-846.

Lemaire G., Salette J., 1982. The effects of temperature and fertilizer nitrogen on the growth of two forage grasses in spring. Grass Forage Sci., 37, 191-198.

Lemaire G., Salette J., Laissus R., 1982. Analyse de la croissance d'une prairie naturelle normande au printemps. I. La production et sa variabilité. Fourrages, 91, 3-16.

Lemaire G., Salette J., Gosse G., Chartier M., 1982. Temperature and spring growth for grasses : comparison of different grass stands. Proc. 9th Gen. Meet. Eur. Grassl. Fed., Reading, 5-9 sept. 1982, Ed. Brit. Grassl. Soc., 255-262.
Payen D., Lemaire G., Salette J., 1982. Variabilité du régime thermique en climat océanique et précocité de croissance de la prairie en fin d'hiver. C.R. Acad. Agric. Fr., 9, 689-699.

Salette J., Dumas Y., 1970. Constantes de comportement de Digitaria decumbens Stent. : Relation entre la teneur en azote et le rapport feuilles/tiges; influence de différentes conditions de milieu. C.R. Acad. Sci., Paris, 271, Série D, 200-202.

Salette J., Lemaire G., 1981. Sur la variation de la teneur en azote de graminées fourragères pendant leur croissance : formulation d'une loi de dilution. C.R. Acad. Sci. Paris, 292, 875-878.

Salette J., Lemaire G., Robichet J., 1982. Nitrogen and mineral uptake during regrowth of pure grass swards. Proc. 9th Gen. Meet. Eur. Grassl. Fed., Reading, 5-9 sept. 1982, Ed. Brit. Grassl. Soc., 165-170.

Thomas H., Stoddart J. L., 1980. Leaf senescence. Annu. Rev. Plant Physiol., 31, 83-111.

Wooledge J., 1978. The effect of shading during vegetative and reproductive growth on the photosynthetic capacity of leaves in a grass sward. Ann. Bot., 42, 1085-1089. 